\title{
AC 2007-1927: A LEARNING PROGRESSION TO EFFECTIVELY IMPLEMENT VIRTUAL REALITY AS AN EDUCATIONAL TOOL FOR K-12 NANOSCIENCE EDUCATION
}

John Bell, University of Illinois-Chicago

Thomas Moher, University of Illinois-Chicago 


\title{
A Learning Progression to Effectively Implement Virtual Reality as an Educational Tool for K-12 Nanoscience Education
}

\begin{abstract}
Nanoscience and engineering are the wave of the future, and nanoscience education is key to developing the next generation of innovative developers. However many K-12 students have difficulty relating to science that they cannot directly observe. Virtual reality based simulations are helpful for exploring these sorts of phenomena, however they must be firmly grounded back to a frame of reference with which students are familiar. This paper presents a progression of learning which uses pixels on a computer screen as a unifying theme to lead students from physical models to microscopic exploration and finally to nanoscale exploration in a virtual world.
\end{abstract}

\section{BACKGROUND}

\section{Nanoscience Education}

Advances in nanoscale science and engineering are occurring at a phenomenal rate, to the extent that many believe that this could be the next big area of human development ${ }^{8}$. The extent of this rapid development is such that soon high school graduates will not be considered "scientifically literate" if they do not have at least a basic understanding of nanoscale phenomena.

Furthermore, in order to train the next generation of nano scientists and engineers, it is important that certain key groundwork be laid down during the K-12 education cycle, so that students are ready to study nanoscience when they enter college. One of these important nanoscale fundamentals is the concept of self-assembly.

\section{Self-Assembly}

The concept of self-assembly involves components that fit together naturally into ordered structures without external action placing them into position, and which retain that ordered structure in the presence of moderate disturbances ${ }^{12,13}$. One common naturally occurring example of self-assembly is protein folding, in which large complex molecules fold themselves into ordered biological structures without an external agent specifically folding and bonding each piece. ( Self-assembly is driven by the attractive and repulsive forces of the atoms in the molecules, as well as stearic hindrance effects that control what portions of what molecules are able to come within close contact, and which are not. )

Modern engineers and scientists have been able to learn from observing these natural processes, and have applied the concepts of self-assembly to the manufacture of microscopic and nanoscopic man-made structures. In particular consider microscopic and nanoscopic electronic components that are too small to be assembled into useful structures using conventional pickand-place assembly methods ${ }^{11,14}$. Self-assembly is an important alternative, in which components are designed to fall into place by themselves, under the influence of attractive and repulsive forces, ( generally hydrodynamics and/or some geometrical configurations. ) One example of this is the pixels on a common LCD computer screen. This latter application is an 
important bridge in this project, providing a crucial connection between macroscopic, microscopic, and nanoscopic environments.

\section{Learning Progression}

It has been found that students often have difficulty learning new material if they do not have any pre-existing frame of reference upon which to relate the new content ${ }^{1}$. Diving straight into totally new and unfamiliar waters leaves them disoriented and confused, with no connections between their previous experiences and the new material being presented. In this case, an underlying framework must be provided, in order to provide some supporting structure for the new material.

Related to this is the concept of learning progression, which refers to the order in which learning takes place ${ }^{10}$. It is important to lay down a solid foundation first, and then to slowly build up a solid reliable path of new material, one carefully laid building block at a time. If any of the important early concepts are skipped, or worse yet learned incorrectly, then there is no point in moving forward until the missing or incorrect learning is corrected.

\section{Design-Based Learning}

All students learn through a wide variety of mechanisms, including reading, lectures, demonstrations, and more active learning methods such as homework and laboratory experimentation. These are all very important, because Dale Edgar found that learners retain more information longer when it is presented through a variety of delivery channels, and the more active the participation the better and longer the information is retained ${ }^{2}$. In addition the concept of learning styles tells us that each learner has a preferred mechanism for learning new material, and if the style of teaching does not match their style of learning, then learning does not take place in an optimal manner ${ }^{3}$.

One mechanism for learning that has been found to be very important is design-based learning, in which students are not handed the solution or even the formulas for getting the solution, but rather are given open-ended design problems that they must work out through experimentation, trial-and-error, and evolutionary design. By making students figure out for themselves what works and what doesn't, ( and more importantly why ), they gain a deeper insight into the underlying concepts of the problem, as well as a better grasp of the big picture and how all the pieces fit together ${ }^{4}$.

\section{Virtual Reality}

Virtual reality, VR, has been used effectively by many researchers as an educational tool, particularly for topics inaccessible in the real world, ( ancient worlds, dangerous environments, or phenomena not normally visible to the human eye. ) 5,7,15 Two obstacles to applying VR to nanoscience at the K-12 level are: (1) Younger students have difficulty making the leap to a scale so small that it cannot be seen, and (2) There needs to be a link relating the familiar physical world to the new and unfamiliar world of VR. This paper presents an approach to lead students from the macroscopic physical world into the nanoscopic virtual world through a series of bridges that provide important educational learning progression. 


\section{PROJECT DESCRIPTION}

Nanoscience education at the K-12 level faces the hurdle that some students have difficulty grasping concepts that they cannot see, or in some cases even believing in their existence. Therefore this project starts by exploring important concepts in self-assembly at the macroscopic scale where they are more easily seen, and then moves to the microscopic and finally nanoscopic worlds. By first exploring microscopic phenomena, students grasp the concept that things too small for them to see really exist nonetheless. From there, the existence of things that are even smaller still, which cannot be seen even with ordinary microscopes, becomes more easily grasped.

\section{Connecting Application}

In order to tie the three worlds together, a common application has been chosen, specifically the placement of pixels on an LCD computer screen. This is just one example of where selfassembly is commonly used in microelectronics, where a large number of very tiny pieces must be assembled into very precise locations. In the case of an LCD screen, a typical $1024 \times 768$ screen contains approximately three-quarters of a million pixels, and three times that many components ( $\sim 2.25$ million elements ) considering that each pixel is composed of a red, green, and blue component. This is far too many elements to be assembled reliably using traditional pick-and-place manufacturing techniques, and so self-assembly is used instead.

In this application, microelectronic components are created on silicon wafers using standard electronics manufacturing techniques, and are then removed from the wafers and placed into a liquid carrier solution. This liquid slurry is then passed over a circuit board or other substrate, and whichever microcomponents pass by their bonding sites with the correct orientation and a suitably slow velocity adhere to the substrate through attractive forces designed into the components and the substrates. Those that do not align properly do not bind, and are recycled with the slurry for another pass ${ }^{6}$. Eventually all of the binding sites are filled, and the process is complete. ( The filled structure may then undergo further processing to more permanently bind the individual components into position. )

The "mating" of microcomponent elements to suitable binding sites may be accomplished through geometrical configurations, ( shaped components that only fit properly into holes with matching shapes ), or through hydrophobic / hydrophilic attractions, ( fluid hydrodynamics ), or through other means. The fluid is generally chosen to give the microcomponents an appropriate approximation to neutral density, and also based on other important fluid properties.

This application should be attractive and interesting to students, because it is something that they can relate to and which they may wonder how it is done. They can also easily relate to the difficulty of placing over 2 million elements in precisely the right locations without a single error.

\section{Physical Models}

The first step on this bridge involves physical models of a computer display screen, with individual "pixels" that need to be placed correctly on the "screen". The figure below shows an early prototype of the physical models, to be refined before project implementation. In the final 
implementation the pixel grids will be $10 \times 10$ ( for practical lessons on percentages ), and the students' goal will be to place all 100 pixels correctly in the grid with the numbers facing right side up and reading correctly.

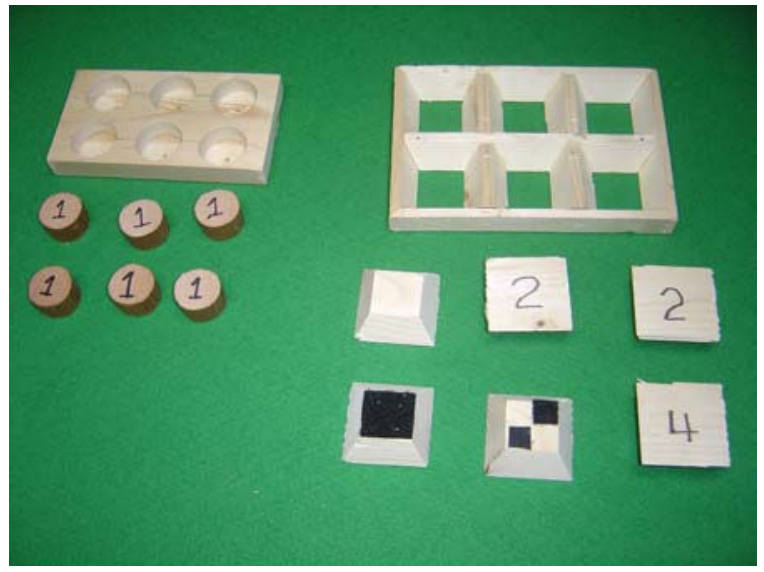

Figure 1 - Prototypes of Physical Models to be Used at the Macro Scale.

In the first iteration of the design, ( pixels labeled " 1 " in the left side of the figure ), each individual pixel must be placed by hand, a tedious and impractical process.

Pixel iteration "2" ( top row right in the above figure ) uses pixels and holes in the shape of truncated square pyramids. The grid will be placed in the bottom of a box, the pixels can be poured in, and the box shaken until as many pixels as possible fall into their proper alignment. The use of a clear-topped box will allow students to see what is happening, and to adjust the style and vigorousness of their shaking. ( Adjusting the energy added to the system. ) They will find that too little shaking does not encourage the pieces to fall into place, and too much causes pixels to jump back out. In the end the students can count how many grid positions received their pixels properly, how many received pixels in an incorrect orientation, and how many did not end up with any pixels at all. Repeating the experiment raises the issues of averages, data analysis, predictability, and repeatability, thereby addressing other educational goals important to $\mathrm{K}-12$ science educators.

Iteration "3" ( bottom row left ) applies Velcro to the bottom of each pixel, which prevents pixels from bouncing back out of the holes once they initially fall into position. With this design evolution all ( or nearly all ) of the holes should eventually get filled, however the orientation of the pixels should only be correct $25 \%$ of the time on average. ( The rest of the " 3 "s will be sideways or upside down. )

Iteration "4" ( bottom row center ) replaces the single piece of Velcro with two smaller pieces, one the "fuzzy" side of the Velcro and one the "hook" side. This adjustment reduces the bonding force to hold pixels into the holes, but ensures that they can only stick into the holes in the correct configuration, and those that do not land properly will be bounced back out again with the shaking of the box. ( This is an important concept in self-assembly, that components may combine briefly in poor configurations, but only the correct configuration has enough binding power to remain bound in the presence of whatever external forces may be present. ) 
Unfortunately, iteration 4 now has a different problem, in that the pixel pieces can stick to each other, and once two pieces stick together they will no longer separate, and can obviously not fit into the holes. A further refinement, ( not shown ), is to go back to the Velcro arrangement of iteration 3, but to "number" the pixels with a set of 5 dots arranged in the pattern on a common die. Since the pattern is now rotationally symmetric, it should be possible to place all of the pixels into their proper position with the right amount and type of shaking of the box.

This succession of constantly improving designs not only illustrates important concepts of selfassembly, but also illustrates the evolutionary process which most good designs undergo, and the fact that the first design conceived is rarely the best. The final iteration, ( converting the roman numeral " 4 " of iteration 4 into a symmetric pattern of five dots for iteration 5 ), illustrates that sometimes the best solution to a difficult design specification, ("the number must be right side up"), is to modify the specifications to be more reasonable and attainable.

\section{Microscopic Exploration}

The second step is to examine a real LCD display unit, using simple affordable microscopes as shown in the figure below. The image on the left shows a low-cost electronic microscope connected to a laptop computer as a display device. The object under the microscope is a piece from a broken LCD computer display, as shown in the right hand image of the figure below.
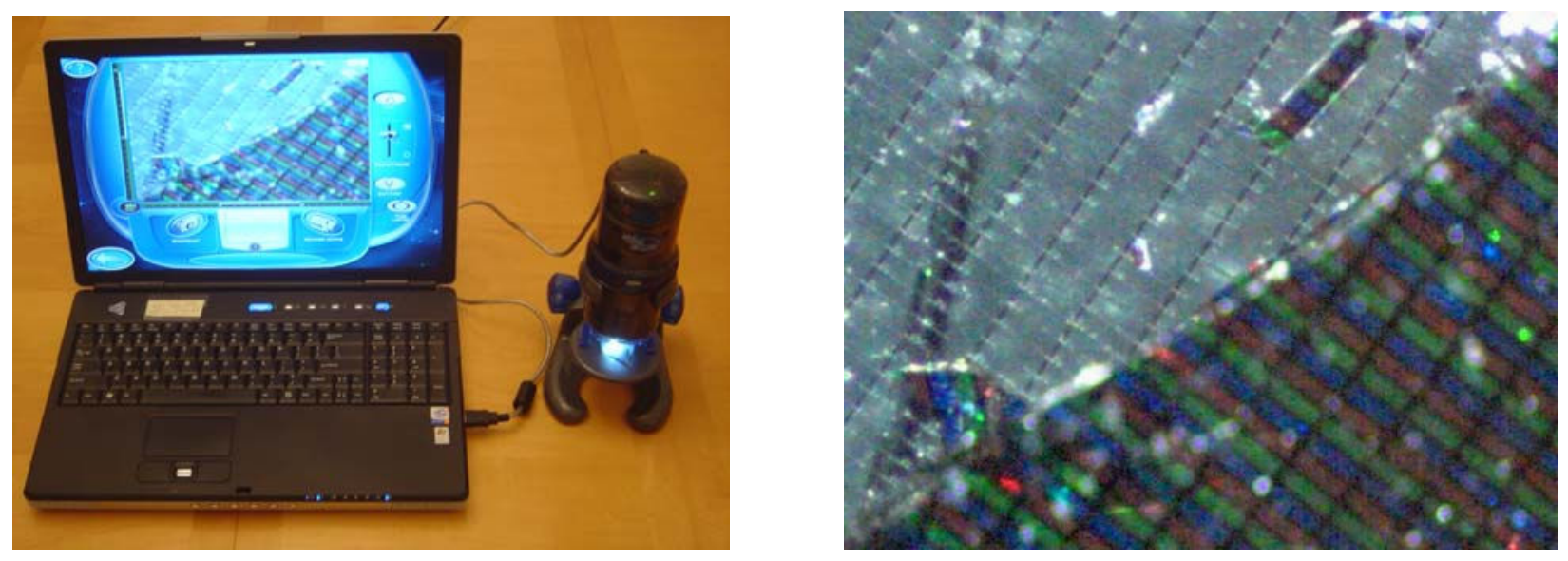

Figure 2 - An Inexpensive USB Microscope Examining a Broken LCD Screen

From this exploration, students learn that the real display does not exactly match the physical models that they worked with earlier, but that it could be assembled using self-assembly techniques. In particular, the LCD pixel elements are rectangular, not square, so that three of them together form a square pixel with red, green, and blue components. ( LCDs act like very small window shutters, either blocking light or allowing it to pass through. Color is provided with a filter of colored glass or plastic that must be aligned perfectly over each LCD shutter. ) The LCDs in the real display also do not exhibit the truncated-pyramid geometry seen in the physical model, although that may be either because the beveled edges are not apparent in the final assembled structure, or there may have been an intermediary stage which was removed after the pixels were bonded permanently into position. It is expected that other manufacturer's panels would appear differently under the microscope, and may more closely match the models. 
Discussions of the real panel design bring up the important concept of reverse-engineering. Students also learn that even although something is too small to be seen with the naked eye, that it exists and has structure nonetheless. From here, the conceptual leap that items still smaller, too small to be seen even with powerful microscopes, can exist and have definite structure as well, is more easily made.

\section{Nanoscale Phenomena in a Virtual World}

The third step is a virtual world, in which the computer pixels seen under the microscope appear as huge gigantic structures. These pixels serve as a connection between the physical world that students have seen in their microscopes and the virtual world in which they can explore nanoscale science and technology. The pixels also serve to set the scale for the virtual world. In some sense, the students are now looking at the computer pixels from "the other side" - that is looking up at them from below instead of looking down on them from above.

The particular topic of study in the nanoscale virtual world will not be locked in to any specific educational topic or learning goal. Rather it will be developed as a general-purpose learning environment where students can explore a wide variety of different educational topics at the nanoscopic scale. It is planned to configure the virtual world so that the actual content material can be read in from data files, thereby allowing a single virtual environment to serve as the background for a wide variety of topic areas, and for new topics to be added without having to rebuild the environment.

Some of the self-assembly related topics that have been considered for initial development include:

- DNA Sequence Matching

- Dielectropheresis
- Protein Folding

- Insulin Production

( In particular it is important to note that in the nanoscopic virtual world the pixels are no longer the subject of principal interest - Rather they form a background framework to put the nanoscale topic of interest in context, and to provide a visual reference of relative scale. )

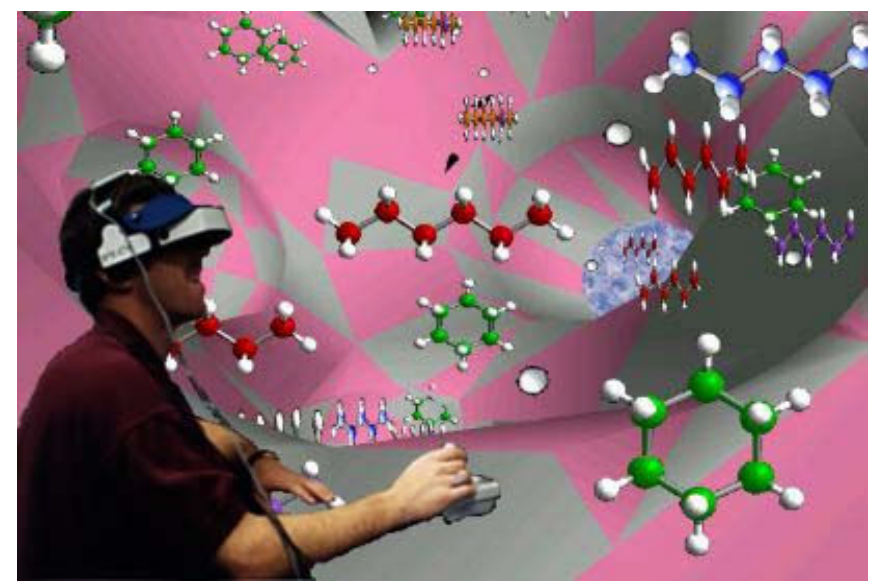

Figure 3 - A Conceptual Rendition of a Student Exploring a Virtual Nanoscopic Environment. 


\section{RELATION TO EDUCATIONAL STANDARDS}

The American Association for the Advancement of Science published a report in 1993 entitled "Benchmarks for Science Literacy" 9 , which outlines what scientifically literate students should be able to do at the end of grades 2, 5, 8, and 12. Some of the important benchmarks outlined in that report that are addressed by the project described in this paper are listed here. (The parenthetical notation indicates the chapter, section, and level of the related standard, where levels are indicated by P for Pre-School benchmarks, E for Elementary School, M for Middle School, and H for High School )

- ( 3 A E ) - "Technology enables scientists and others to observe things that are too small or too far away to be seen without them and to study the motion of objects that are moving very rapidly or are hardly moving at all."

- ( 4 D E ) - "With magnifiers, students should inspect substances composed of large collections of particles, ..., to discover the unexpected details at smaller scales."

- ( $8 \mathrm{~B} \mathrm{H}$ ) - "Increased knowledge of the molecular structure of materials helps in the design and synthesis of new materials for special purposes."

- $(8 \mathrm{E} \mathrm{H})$ - "Miniaturization of information-processing hardware can increase processing speed and portability, reduce energy use, and lower cost. Miniaturization is made possible through higher-purity materials and more precise fabrication technology."

- (11 B M ) - "Models are often used to think about processes that happen too slowly, too quickly, or on too small a scale to observe directly, or that are too vast to be changed deliberately, or that are potentially dangerous."

- $(11 \mathrm{C} \mathrm{H}$ ) - "A system in equilibrium may return to the same state of equilibrium if the disturbances it experiences are small. But large disturbances may cause it to escape that equilibrium and eventually settle into some other state of equilibrium."

- (11 D H ) - "Because different properties are not affected to the same degree by changes in scale, large changes in scale typically change the way that things work in physical, biological, or social systems."

Initial implementation of this program is most likely to take place at the high school level. A single class period should be sufficient to go through both the physical models and the microscopic exploration, plus one or more additional periods to explore the virtual world, depending on the complexity of the specific topics studied. In order to promote discussion of the topics, this activity is best conducted in groups of 3 to 4 students.

\section{CONCLUSION}

A method has been presented for the delivery of nanoscience education that incorporates a real world example as a common thread to connect the learning progression from the macroscopic through the microscopic to the nanoscopic world. Specifically the placement of pixels on an LCD computer screen has been proposed as a unifying theme to lead students from macroscopic physical models, ( where they learn important concepts of self-assembly and evolutionary design ), through microscopic examination, ( where they learn about reverse-engineering and the fact that models are not always completely accurate ), and into a virtual-reality based learning environment where microscopic pixels appear the size of large buildings and nanoscale 
phenomena can be explored in context. The proposed approach is based upon solid pedagogical foundations, and has been connected to related standards of scientific literacy.

\section{ACKNOWLEDGEMENTS}

The author gratefully acknowledges the support of this research by The National Science Foundation under Grant No 0426328, as part of the ongoing work of the National Center for Learning and Teaching in Nanoscale Science and Engineering ( NCLT ). Thanks are also given to Tom Moher, Jim Pellegrino, Carmen Lilley, Andy Johnson, Brenda Lopez, Marco Bernasconi, Don Olmstead, Shanna Daly, Florencia Anggoro, and Emily Shipley for their feedback and other support for this work, and to the University of Illinois at Chicago for their support.

\section{BIBLIOGRAPHY}

1. Bransford, J., Brown, A., and Cocking, R., "How People Learn". Washington, DC: National Academy Press, 1999.

2. $\quad$ Edgar, D., "Audio-Visual Methods in Teaching", Third ed: Holt, Rinehart, and Winston, 1969.

3. Felder, R. M. and Silverman, L. K., "Learning and Teaching Styles in Engineering Education", Journal of Engineering Education, vol. 78, pp. 674-681, 1988.

4. Kolodner, J. L., Camp, P. J., Crismond, D., Fasse, B., Gray, J., Holbrook, J., and Puntembakar, S., "Problem-Based Learning Meets Case-Based Reasoning in the Middle-School Science Classroom: Putting Learning-by-Design into Practice", Journal of the Learning Sciences, vol. 12, pp. 495-548, 2003.

5. Loftin, R. B., Engelberg, M., and Benedetti, R., "Applying Virtual Reality in Education: A Prototypical Virtual Physics Laboratory", IEEE Symposium on Research Frontiers in Virtual Reality, Los Alamitos, CA, 1993.

6. Morris, C. J., Stauth, S. A., and Parviz, B. A., "Self-Assembly for Microscale and Nanoscale Packaging: Steps Toward Self-Packaging", IEEE Transactions on Advanced Packaging, vol. 28, pp. 600-611, 2005.

7. Pantelidis, V. S., "Virtual Reality and Education: Information Sources; A Bibliography", http://www.coe.ecu.edu/vr/vpbib.html,

8. Roco, M. C., "From Vision to the Implementation of the U.S. National Nanotechnology Initiative", Journal of Nanoparticle Research, vol. 3, pp. 5-11, 2001.

9. $\quad$ Science, A. A. f. t. A. o., "Benchmarks for Science Literacy": Oxford University Press, 1993.

10. Smith, C., Wiser, M., Anderson, C., and Krajcik, J., "Implications of Research on Children's Learning for Standards and Assessment: A proposed Learning Progression for Matter and Atomic-Molecular Theory", Measurement, vol. 14, pp. 1-98, 2006.

11. Verma, A. K., A.Hadley, M., Yeh, H.-J. J., and Smith, J. S., "Fluidic Self-Assembly of Silicon Microstructures", Proceedings of the 45th Electronic Components and Technology Conference, pp. 12631268, 1995.

12. Whitesides, G. M. and Boncheva, M., "Beyond Molecules: Self-Assembly of Mesoscopic and Macroscopic Components", PNAS, vol. 99, pp. 4769-4774, 2002.

13. Whitesides, G. M. and Grzybowski, P., "Self-Assembly at All Scales", Science, vol. 295, pp. 2418-2421, 2002.

14. Xiong, X., Hanein, Y., Fang, J., Wang, Y., Wang, W., Schwartz, D. T., and F.Bohringer, K., "Controlled Multibatch Self-Assembly of Microdevices", Journal of Microelectromechanical Systems, vol. 12, 2003.

15. Youngblut, C., "Educational Uses of Virtual Reality Technology", Technical Report IDA Document D$2128,1998$. 